\title{
Guillain-Barré Syndrome and SIADH in a Patient With Chronic Lymphocytic Leukemia
}

David R. Gutknecht, MD

Guillain-Barré syndrome, an acute or subacute inflammatory demyelinating polyradiculoneuropathy, usually causes ascending paralysis, areflexia, and sensory disturbances. ${ }^{1} \mathrm{~A}$ similar syndrome, more chronic and sometimes relapsing, has been called chronic inflammatory demyelinating polyneuropathy. ${ }^{2}$ Thought to have an immunologic cause, Guillain-Barré syndrome can be found in association with infections, vaccinations, and malignancies. The syndrome has been linked to carcinomas and to Hodgkin and non-Hodgkin lymphomas, and both Guillain-Barré syndrome and chronic inflammatory demyelinating polyneuropathy have been seen with the chemotherapy and other interventions associated with bone marrow transplantation. ${ }^{3}$ Guillain-Barré syndrome has, however, only rarely been reported in patients with chronic lymphocytic leukemia. ${ }^{4,5}$ We report the occurrence of a painful, acute polyneuropathy, with features of Guillain-Barré syndrome and complicated by the syndrome of inappropriate antidiuretic hormone secretion, which affected an elderly woman who underwent chemotherapy for chronic lymphocytic leukemia.

\section{Case Report}

An 84-year-old woman with a 6-year history of untreated chronic lymphocytic leukemia complained of several years of mild tingling of her fingers and toes. Her lower extremity reflexes were absent, and she had decreased but preserved upper extremity reflexes, and decreased proprioceptive and vibratory sensation in her legs. There was no weakness, and serum levels of folate and vitamin $B_{12}$ were normal. Findings on magnetic resonance imaging of the entire spine were unremarkable except for an abnormal marrow signal consistent with leukemic infiltration. Electromyography and cerebrospinal fluid examination were not per-

Submitted 23 July 1997.

From the Department of General Internal Medicine, Geisinger Medical Center, Danville, Pa. Address correspondence and reprint requests to David $R$. Gutknecht, $M D$, Geisinger Medical Center, Danville, PA 17822. formed. The white cell count was $92,000 / \mu \mathrm{L}$ with 90 percent lymphocytes. Flow cytometry showed a monoclonal B cell population consistent with chronic lymphocytic leukemia. Quantitative serum immunoglobulin levels were normal, and there was no monoclonal gammopathy. She received her first, and only, course of chemotherapy in the form of prednisone, $150 \mathrm{mg}$ daily, and concurrent chlorambucil, $22 \mathrm{mg}$ daily, for 5 days, and her white cell count declined to $60,000 / \mu \mathrm{L}$.

During the week following her chemotherapy, she complained of an aching pain in the mid and lower back. She was hospitalized, and when examined, she had residual cervical and axillary adenopathy, her blood pressure was $174 / 69 \mathrm{mmHg}$, and her strength was normal. There were no abnormal findings during a back examination. Her blood pressure thereafter rose as high as 199/76 $\mathrm{mmHg}$, and she complained of urine retention. A computed tomographic (CT) scan of her pelvis showed a distended urinary bladder and a reduction in previously documented pelvic adenopathy. On the evening of the 3 rd hospital day, her pain disappeared, but she had trouble using her hands to eat. On the 4th day, limb weakness was clearly evident. She again had no reflexes in her legs, but strength in her legs was now grade 2/5 and in the arms grade $3 / 5$, with loss of reflexes in the arms. There was no respiratory distress and no involvement of cranial nerves.

Results of a CT examination of her brain were normal, and a porphyria screening test was negative. Cerebrospinal fluid examination findings were glucose $77 \mathrm{mg} / \mathrm{dL}$, protein $64 \mathrm{mg} / \mathrm{dL}$, and 3 round cells per microliter. Findings of a cytologic examination of cerebrospinal fluid were negative. An electromyogram showed delayed distal latencies and prolonged $F$ waves consistent with Guillain-Barré syndrome. Plasma exchanges, of approximately $2000 \mathrm{~mL}$ each, were given daily for 5 days.

Her serum sodium, $138 \mathrm{mEq} / \mathrm{L}$ on admission, was $117 \mathrm{mEq} / \mathrm{L}$ on the morning before the first plasma exchange. Her serum osmolality was 245 
$\mathrm{mOsm} / \mathrm{L}$, with urine osmolality $711 \mathrm{mOsm} / \mathrm{L}$, and urine sodium $22 \mathrm{mEq} / \mathrm{L}$. The patient's state of hydration and results of her thyroid tests and serum creatinine levels were normal. She was not taking diuretics or drugs known to stimulate antidiuretic hormone release. Fluid restriction was begun, and her serum sodium levels increased to normal.

After plasma exchange the patient gradually recovered full power in her arms, and the strength in her legs improved considerably. At no time were there respiratory or cranial nerve problems, although periodic bladder catheterization remained necessary. She was transferred to a rehabilitation facility on the 23 rd hospital day.

\section{Discussion}

The patient had chronic lymphocytic leukemia and a mild chronic sensory neuropathy. She then suffered a subacute illness that caused pain, ascending paralysis, loss of arm reflexes, and hyponatremia. Cerebrospinal fluid and electromyographic findings at the time of this subacute syndrome were consistent with those of GuillainBarré syndrome; and although it was not possible to know whether these findings were new or old, because baseline studies had not been done before the onset of paralysis, we have chosen to consider her illness Guillain-Barré syndrome because of its clinical presentation. Paralysis rarely progresses at such a pace in chronic inflammatory demyelinating polyneuropathy. ${ }^{2}$

Why a patient with chronic lymphocytic leukemia developed Guillain-Barré syndrome is not clear. The association might have been coincidental. There was, however, no antecedent vaccination, infection, or history suggestive of Campylobacter jejuni enteritis, and both Guillain-Barré syndrome and chronic lymphocytic leukemia are disorders in which disruptions of the immune system occur. Powles and Malpas ${ }^{4}$ thought the disorders might be linked through the greater susceptibility to viral infection found with the antibody deficiencies of chronic lymphocytic leukemia. Jackson, ${ }^{5}$ on the other hand, suggested that immunosuppression from chemotherapy could have played a role in the case he reported. The patient we report received chlorambucil just 10 days before her paralysis began, but a direct connection between that treatment and her neurologic illness cannot be proved.

The hyponatremia, which complicated this case, appeared to be caused by the syndrome of inappropriate secretion of antidiuretic hormone, or SIADH. An association of SIADH with GuillainBarré syndrome is well-established and commonly noted in review articles, although detailed reports are few. ${ }^{6,7}$ Why a peripheral nerve disorder should trigger antidiuretic hormone secretion is not clear, but in some of the patients reported, antidiuretic hormone release might have been due to the autonomic instability so common in Guillain-Barré syndrome. Autonomic afferent impulses from stretch receptors in the left atrium and carotid body are known to modulate antidiuretic hormone secretion, and failure of such vagal feedback could lead to free water retention. ${ }^{6}$ Hochman et al, ${ }^{7}$ however, reported SIADH in a patient with Guillain-Barré syndrome who had no autonomic dysfunction, and the autonomic system abnormalities in the patient we report were limited to minor blood pressure fluctuations and urine retention. Whatever the causal link, SIADH can be a major complication in Guillain-Barré syndrome and can in some patients cause hyponatremic seizures and even coma. Fortunately, our patient suffered no such problems and had a prolonged but uneventful recovery.

One element of her experience, however, does deserve further comment. Pain is more common in Guillain-Barré syndrome than many clinicians think. Ropper and Shahani ${ }^{8}$ reported deep aching pain in the back, buttock, or upper legs in 55 percent of the patients in their series. Pain was often worse at night and sometimes preceded weakness by up to 5 days. Our patient had similar difficulty, and it was only when paralysis ensued that the cause of her pain became clear.

\section{References}

1. Ropper AH. The Guillain-Barré syndrome. $\mathrm{N}$ Engl J Med 1992;326:1130-6.

2. McCombe PA, Pollard JD, McLeod JG. Chronic inflammatory demyelinating polyradiculoneuropathy. A clinical and electrophysiological study of 92 cases. Brain 1987;110(Pt 6):1617-30.

3. Mudad R, Hussein A, Peters WP. Guillain-Barré syndrome following autologous bone marrow transplantation. Am J Clin Oncol 1995;18:167-9.

4. Powles RL, Malpas JS. Guillain-Barré syndrome associated with chronic lymphatic leukaemia. Br Med J 1967;3:286-7.

5. Jackson M. Guillain-Barré syndrome in a patient with chronic lymphocytic leukaemia. Postgrad Med 
J 1993;69:832-3.

6. Davies AG. Inappropriate secretion of antidiuretic hormone in Guillain-Barré syndrome. Postgrad Med J 1971;47:651-3.

7. Hochman MS, Kobetz SA, Handwerker JV. Inap- propriate secretion of antidiuretic hormone associated with Guillain-Barré syndrome. Ann Neurol 1982;11:322-3.

8. Ropper AH, Shahani BT: Pain in Guillain-Barré syndrome. Arch Neurol 1984;41:511-4. 